\title{
The Development of a Prototype of Bionic Eyes for Visual Impairment
}

\author{
Ponglert Rattanachinalai ${ }^{\mathrm{a}^{*}}$, Wanglok Do ${ }^{\mathrm{b}}$, Soranut Kittipanyangam ${ }^{\mathrm{c}}$, Kei Eguchi ${ }^{\mathrm{d}}$ \\ a,b,c,dFukuoka Institute of Technology, 3-30-1 Wajiro Higashi, \\ Higashi Ward, Fukuoka, 811-0295, Japan
}

Corresponding Author: aponglert.r@gmail.com, bdw112345@ naver.com, ${ }^{\mathrm{c}}$ soranutac123@gmail.com, ${ }^{\mathrm{d}}$ eguti@ fit.ac.jp

\begin{abstract}
Nowadays, people who have visual impairment like blindness which make them completely lost there's vision, they may have a lot of things to help them in daily life. Example; Braille, White cane, Foot path sign and others. However, there is some reason those item is not enough for them. Many researchers develop Visual Prosthesis that can restore the lost vision of blind people, but those device are too large. Some of it is not portable. And uses complex components.

In this paper, we design and develop the prototype of Visual Prosthesis named Bionic Eyes. Bionic Eyes consists of two parts Smart Glasses and Artificial Eye. The first part of system is Smart Glasses that can be built from a tiny computer or microprocessor. The second part is Artificial Eye that can be built from small circuit that has the same size as eye ball. We can proceed through a lot of process like image processing easily by using Intel Edison as our main processor unit for Smart Glasses.
\end{abstract}

Keywords: Visual Prosthesis, Visual Impairment, Edge Detection, Image Processing.

\section{Introduction}

Blind people have a lot of inconvenient in their life ${ }^{(1)}$. Walking, eating, working, or something like these, there are normal people can doing it easily, but blind people cannot do it as us. When people become blind or blind from birth, they gained other superior sensing ability of listening or touching things in return. They can hear a sound better than normal people. However they have those superior sensing abilities, it's still not enough for them. The ability in vision is incomparable. In order to restore blind people's lost vision, researcher had developed Visual prosthesis.

Previous Visual prosthesis that have been developed by others researcher was used many complex components that make the size of visual prosthesis too large. It may cost a lot of money to build just 1 device for blind people. When the size of Visual prosthesis is too large, it could become inconvenient to use in everyday life of blind people. Image that blind people can see from using those Visual prosthesis may not be perfectly clear, because methods of reducing image's information to easily transmit were still not good enough, and some of the method to restore the lost vision of patients looks dangerous for human's brain.

To solve the problem about previous Visual prosthesis, we designed and developed a prototype Visual Prosthesis named Bionic Eyes. Bionic Eyes consists of two parts: Smart Glasses and Artificial Eye. We divided Bionic Eyes into two parts of system because the area for eyeball on human's body have limitation. Smart Glasses will use image processing on main processor unit to extract necessary information from image, resize image and convert image into binary text file to transmit to the Artificial Eye. Image processing algorithm that we used is Edge Detection method. This method will extract necessary information of image into black and white image. This black and white image have a good information on it that we can easily understand what is in the image better than the previous Visual Prosthesis.

Smart Glasses could easily build with one tiny computer called Intel Edison. Intel Edison is our main processor unit for Smart Glasses. Intel Edison have a necessary function like embedded Wi-Fi and Bluetooth,

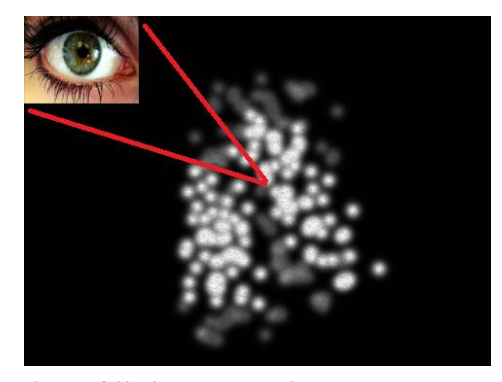

Fig. 1. The "dot of light" cause by Phosphene phenomenon that human's eye can see 
embedded memory and RAM. Intel Edison which is as small as a stamp. It mean that our Visual Prosthesis system size will not become too large like the others. Inside of Artificial Eyes that will be a replacement for human's eye ball, have a necessary small circuit for communicating with Smart Glasses, creating current neural signal and transmission to human's optic nerve. With these method it will not be harmful to human's brain like others method too.

\section{Research Survey}

In the present, methods of using Visual Prosthesis to restore lost vision of patient have 3 main methods.

(a) Stimulus of Visual Cortex

This method is an idea that uses the device sending signal wave to visual cortex of human's brain cerebral cortex that is responsible for processing visual information, or using microelectrode arrays (MEAs) direct penetrating to human's visual cortex and generating a "dot of light" called Phosphene Phenomenon by stimulus the brain's visual cortex (2). As shown in Fig. 1, it is the Phosphene Phenomenon.

\section{(b) Artificial Retina}

This method is development of an electronic visual prosthesis that works as human eye's photoreceptor or retina. It can implant or replace into damaged part in the human's eye. Artificial Retina can create the loss vision by electrically activating eye's cell in human's visual system from

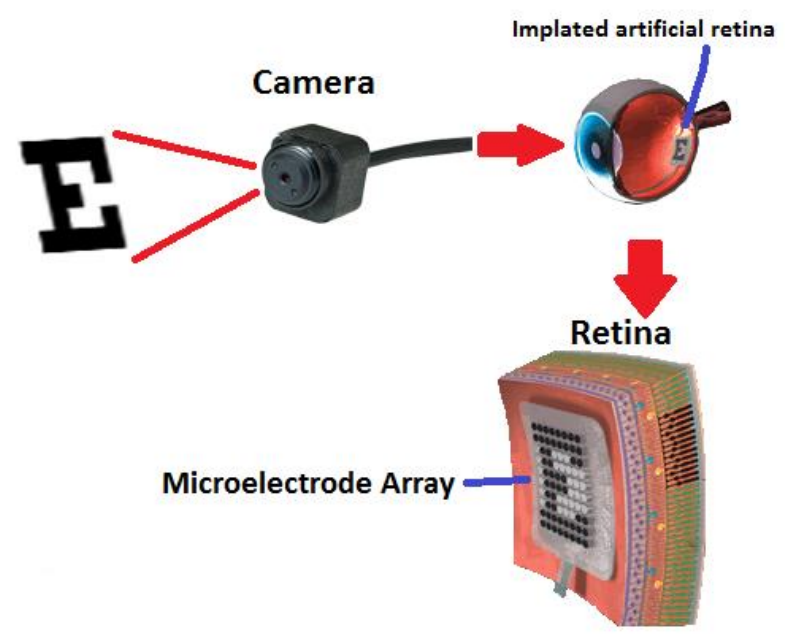

Fig. 2. Artificial Retina concept. Captured image by camera, and send image information data to implanted Artificial Retina, Artificial Retina will stimulate the retina in a pattern of electricity by using Microelectrode Array. By stimulate this eye's part the eye will work like normal again. implanted Artificial Retina that had neural stimulator to create electrical simulation ${ }^{(3)}$. As shown in Fig. 2, it is the concept about Artificial Retina.

(c) Stimulus of Optic Nerve

This method is using camera to capture image, convert image to biphasic current pulse that work like human's optic neural signal and directly send to human's optic nerve by using microelectrode arrays penetrate to human's optic nerve. As shown in Fig. 3, it is kind of the microelectrode arrays that use in many research about activity of human's and animal's nerve. Human's optic nerve is the nearly possible pathway to success restore vision loss, because human's optic nerve is part of human's body that It has purpose to transmit visual information data from human's eye retina to brain with optic neural signal ${ }^{(4)}$.

On the previous research of Visual Prosthesis, almost of it base on using Stimulus of Visual Cortex and Artificial Retina. However our Bionic Eyes will develop under stimulus of optic nerve. Stimulus of visual cortex method have a risk, when the human's brain taking an operation to transplant receiver IC chip, or microelectrode array, it will get damage from operation or malfunction of IC chip. In some cases blind patients who lost their eyeball. It means that they do not have retina. This mean Artificial Retina method cannot be using on this case. Method of using stimulus of optic nerve will be a good method, because it will not harm human's brain and can be use even patients do not have eyeball. The previous Visual Prosthesis developed with complex system that provided too much information or some of it provided less information for patient. Some of previous one was look bulky and feel uncomfortable to use in everyday life. Our Smart Glasses of Bionic Eyes part built from tiny powerful computer that can provide us future benefit function that it will be add in future. Artificial Eye of Bionic Eyes part will be built to provide work load of system not like the others Visual Prosthesis that was not have this part. In Future research Bionic Eyes system built into one system on Artificial Eyes.

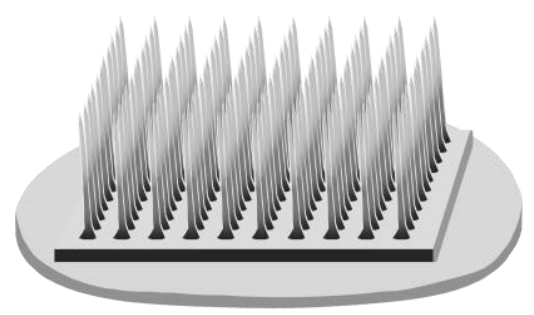

Fig. 3. Layout picture of Microelectrode Array that used in research about neural activity 

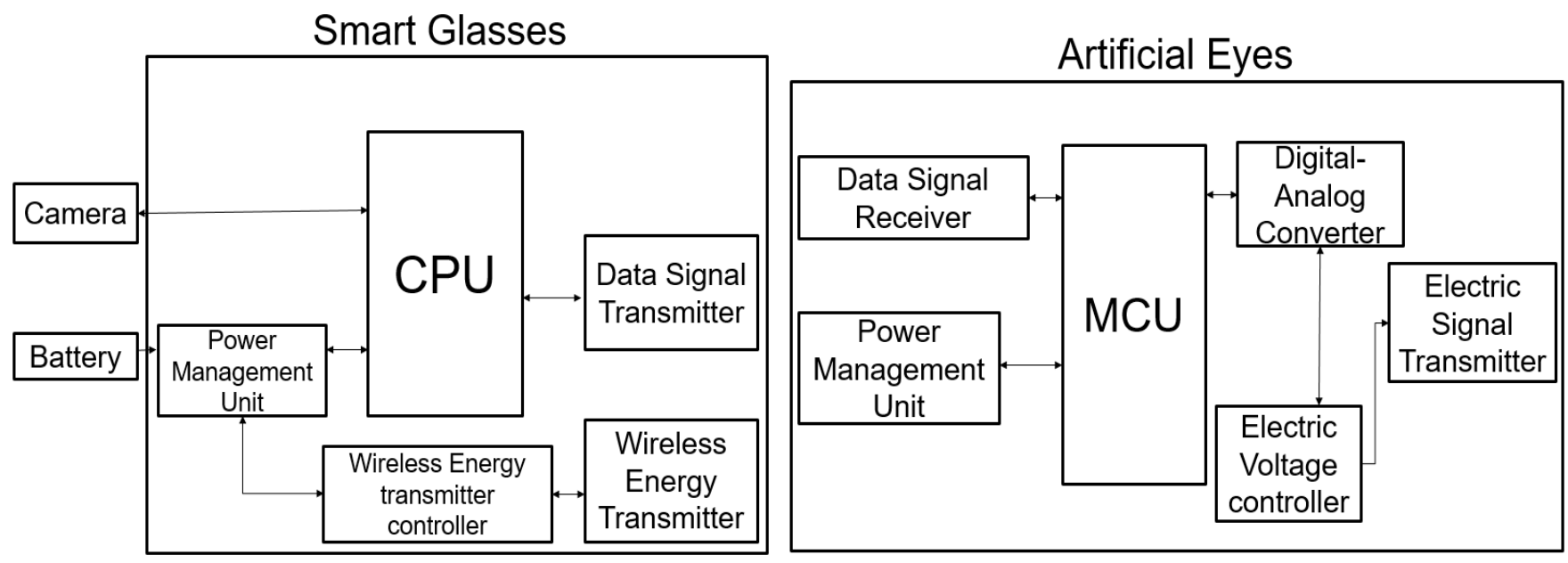

Fig. 4 Block diagram of Bionic Eye's system,

first part is Smart Glasses and second part is Artificial Eyes

Visual Prosthesis system want. Therefore, the other part of Bionic Eye will develop with small necessary circuit too.

\section{Bionic Eye System Structure}

Our research designs and develops Visual Prosthesis's system for people who have complete loss vision from accidents or deceases. Because people who had complete loss vision from birth, they do not have image information memory or experience about shape or color of the things that they cannot see. Our research was focus on Stimulus of Optic Nerve method. We designed and developed Prototype Visual Prosthesis's system that we named Bionic Eye.

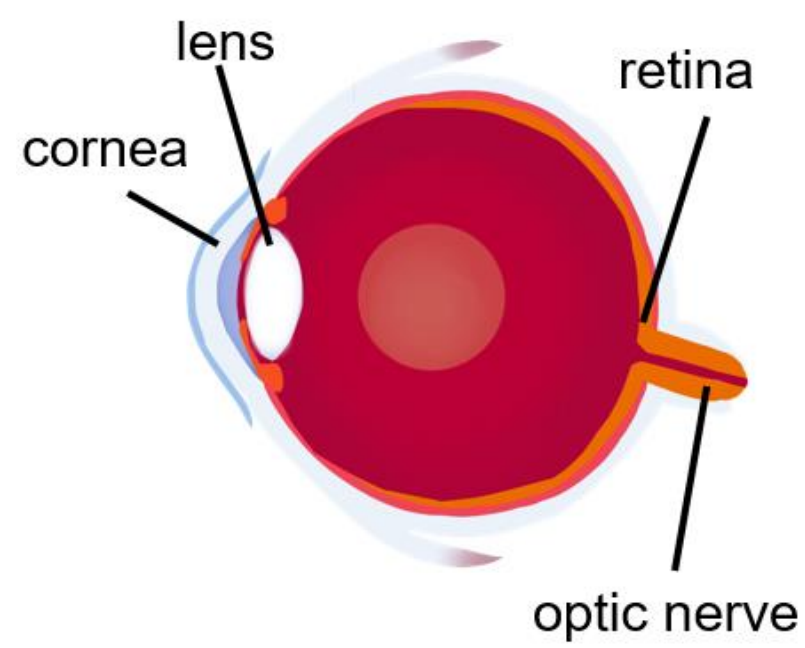

Fig. 5. Human's eye anatomy (a) Human 's eye

Human's eye have many parts that working together to make human can see things. As shown in Fig. 5, it is the human's eye anatomy. Mechanism of the normal camera that we use is work like human's eye mechanism. Camera lens working as eye's cornea, use to focus a light that reflects to the eye. Auto focus function of camera works as eye's crystalline lens along the cornea. Sensor of digital camera works as eye's retina which has purpose to convert light (image) into electric signal and transmit the electric signal to brain's visual cortex by optic nerve. This all mean some hint that we can design and develop a replacement device calling visual prosthesis to restore the loss vision for people who lost it.

(b) Bionic Eye

Prototype Bionic Eyes that we designed were divided into two parts of system working together, as shown in Fig. 7, it is a block diagram of Bionic Eye's system. We divided Bionic Eyes system into two parts, because inside of human's skull has limitation of area for an eye ball. Present technology powerful microprocessor which can fit inside human skull's eye ball's location will have some problems too. If we fuse everything we need into it.

The First part of Bionic Eye's system is Smart Glasses that shown in Fig. 6. Prototype Smart Glasses, it had camera attached at front of the glasses. This camera was connected to main processor unit. The purpose of this camera is, to capture image's information and transmit it to main processor unit. At first stage of our research wanted to use 
the image's information that was easy to understand and did not too large for high speed conversion and transmission. As shown in Fig. 6, it is processing flow chart on main processor unit. The main processor unit took duty to received image from camera, then it used image processing to extract an important information of captured image by image processing algorithm called Edge Detection algorithm. After the main processor unit extracted the important information of captured image to Edge Detection's image, the main processor unit will resize the Edge Detection's image from original size resolution $480 \times 360$ pixel to small size resolution $25 \times 25$ pixel, and convert resized

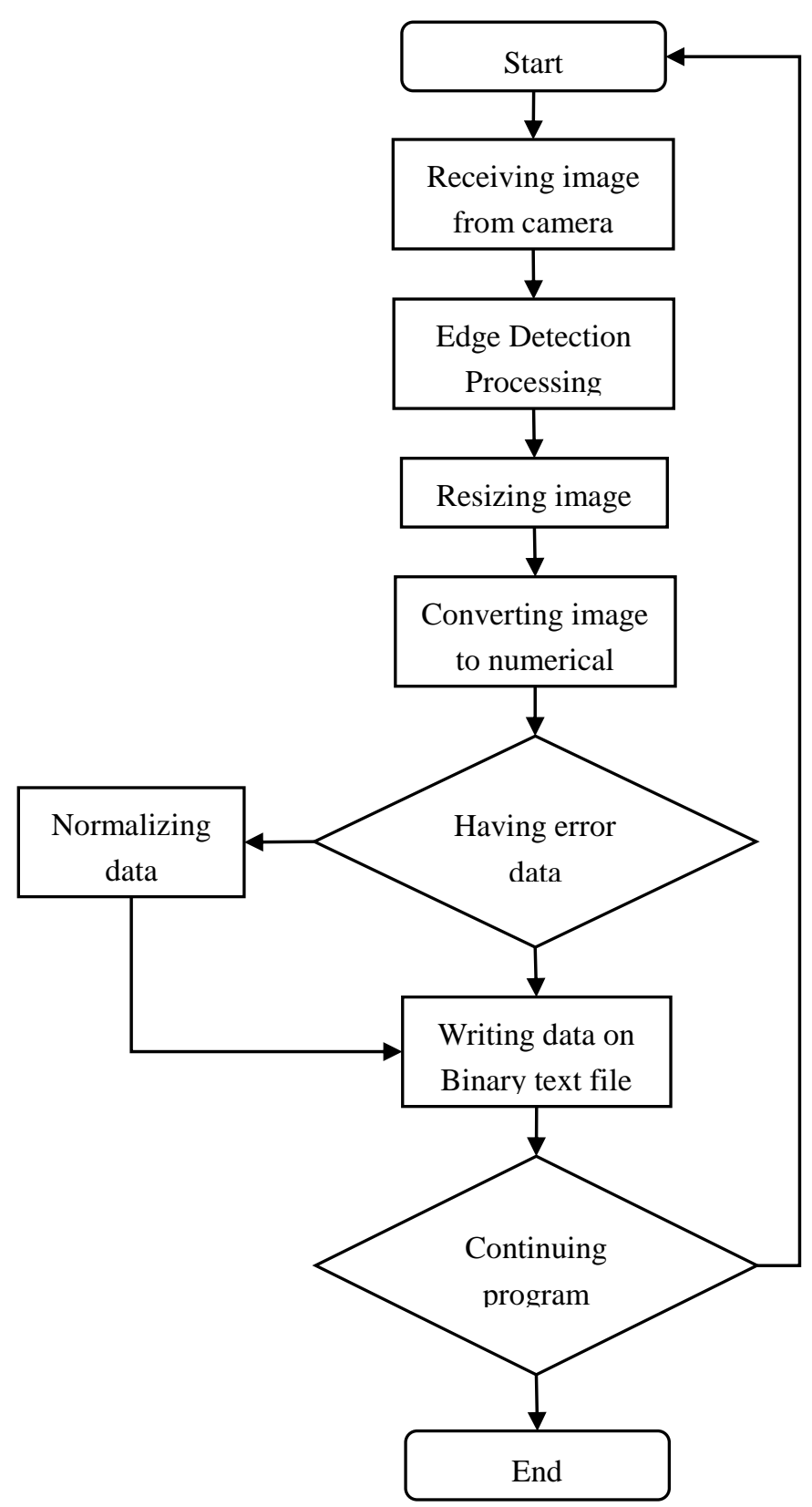

Fig. 6. Flow chart of Image Processing on Intel Edison
Edge Detection's image to binary text file by using image processing. Main processor unit converted the resized Edge Detection's image into binary text file for designing and developing electric current signal. It work like neural signal transmission from retina to optic nerve. And the last process on main processor unit is transmission information data on binary text file to the second part of Bionic Eye's system.

The Second part of Bionic Eye's system is Artificial Eye. Artificial Eye is a replacement eye that will transplant to human body instead of an old one that unable to use. This Artificial Eye will have an integrated circuits inside, which will work together with the Smart Glasses. Artificial Eye will receive transmitted information data, and use it to create digital electric current signal. After it was creating digital electric current signal, Artificial Eye will use integrated digital-analog converter circuit to transform created digital electric current signal to an analog electric current signal. When Artificial Eye had the analog electric current signal data that can transmit to optic nerve through Microelectrode Array which connected from integrated circuit of Artificial Eye, Artificial Eye will use an integrated current controller circuit to transmit the analog electric current signal to optic nerve. To avoid human's optic nerve get damage, this integrated current controller circuit have to control output current signal that the output current will be not burden over for human's optic nerve physical limitations, which may cause from error of Artificial Eye's system or integrated circuit. This Artificial Eye need cooperation from doctor who can transplant Electronic Device as this Artificial Eye on patient, because Artificial Eye has Microelectrode Array which need to direct penetrate to optic nerve.

About Bionic Eye's energy source, the Smart Glasses main processor unit has its own energy source. Our design used rechargeable lithium-ion battery as energy source for main processor unit on Smart Glasses. This Lithium-ion need to have an energy capacitor that fulfill working duration time of Smart Glasses at least 12 hours, because 12 hours is time which human still wake. The recharging system of Smart Glasses will use wireless charging and can use charging cable upon user flavor. Smart Glasses will have a wireless energy transmitter. The wireless energy transmitter will transmit energy to Artificial Eye all time of working. Furthermore about Artificial Eye energy source, Artificial Eye will not have its own main power source inside. That why the Smart Glasses need to have wireless energy transmitter to transmit energy to Artificial Eye. Because if Artificial Eye transplanted into human body have its own energy source like some kind of battery when energy source 


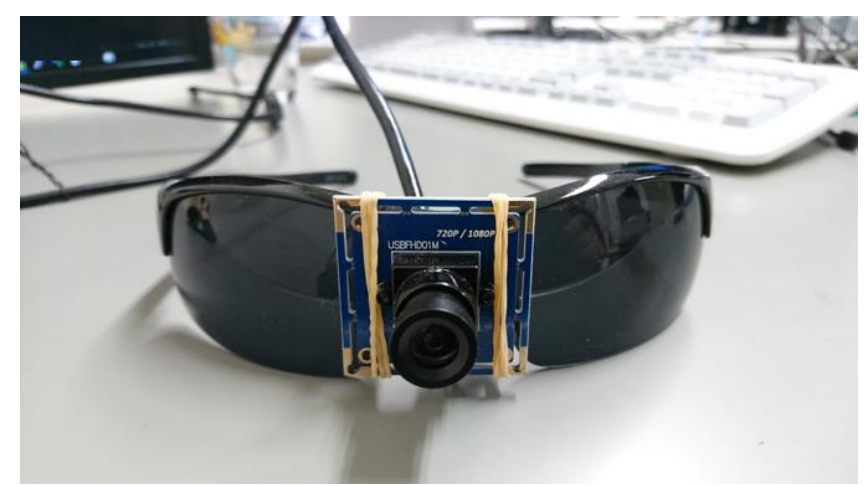

Fig. 7. Prototype Smart Glasses

have malfunction like explode or have chemical compound come out from it. This kind of accident of malfunction will damage the patient who had transplanted Artificial Eye. To avoid every unexpected accidents cause from energy source inside Artificial Eye, we designed Artificial Eye that must receive transmitted energy from Smart Glasses. When Artificial Eye receive transmitted energy, it will collect some energy to super capacitor. Artificial Eye's system can working by using external energy source as main energy. If Artificial Eye cannot use external energy source, it will use energy from supercapacitor as back up energy for short period of time.

(c) Human's optic nerve physiological parameter

Claude Veraart et al, there had many research results about human's optic nerve physiological parameter or about physical limitations. A based parameter for Visual Prosthesis need to use on optic nerve are biphasic current pulses, amplitude from micro-ampere to milliampere and pulse duration in microsecond unit ${ }^{(4)(5)(6)}$.

\section{Experiments}

\subsection{Hardware}

We started our Prototype Bionic Eyes developed, from first part of Bionic Eyes that is Smart Glasses.

(a) Main Processor Unit

We chose Intel Edison for our main processor unit on Smart Glasses. Intel Edison is a tiny computer which Intel offered developed this system for wearable device. As shown in Fig. 8, Intel Edison have a part that most need to be a stand-alone computer. On Intel Edison's board has its own integrated central processor unit (CPU) Intel Atom Processor (dual core $500 \mathrm{MHz}$ ), random access memory (ram) capacity 1 Gigabytes LPDDR3, embedded MultiMediaCard (eMMC) capacity 4 Gigabytes working as hard disk for Intel Edison.
Table 1. Microcontroller and Intel Edison specification

\begin{tabular}{|c|c|c|}
\multicolumn{3}{|c}{ comparison. } \\
\hline CPU clock speed & Intel Edison & Rasberry Pi B+ \\
\hline RAM & $1 \mathrm{~GB}$ & $700 \mathrm{Mhz}$ \\
\hline Memory & $4 \mathrm{~GB}$ & $\begin{array}{c}\text { Need to buy SDcard } \\
\text { to install on board }\end{array}$ \\
\hline WI-Fi & Dual band 802.11 & Do not have \\
\hline Bluetooth & $2.1 / 4.0$ & Do not have \\
\hline Power Input & 3.15 to $4.5 \mathrm{~V}$ & $5 \mathrm{~V}$ \\
\hline Price & 43.9 USD & $53.38 \mathrm{USD}$ \\
\hline
\end{tabular}

Furthermore, The Intel Edison is has embedded Wi-Fi and Bluetooth and USB2.0 controller. All of that part was integrated on tiny chip Intel Edison, which have size about 25 x 35.5 millimeter ${ }^{(10)}$. Intel Edison operating system is Yocto project. It is custom LINUX-based system for embedded chipset or microcontroller. In table 1 , it is a comparison of example of others microcontroller and Intel Edison.

(b) Camera

We used small UVC CMOS camera. Size of the camera is 32 x 32 millimeter, 12 millimeter lens, Max resolution 1920 x 1080 pixel. Camera frame rate about 60 frame per second. This UVC COMOS camera was connected to Intel Edison by USB2.0 port. We chose this UVC camera because its low cost and specification of camera enough for the prototype device.

(d) Power source

In our research, we need to use battery to make our main processor unit to be carry-on device. When our main processor unit can carry to other place and work that mean we can have an experiment at anywhere, and test the duration working time of the battery. We installed lithium-ion battery on main processor unit through developer board.

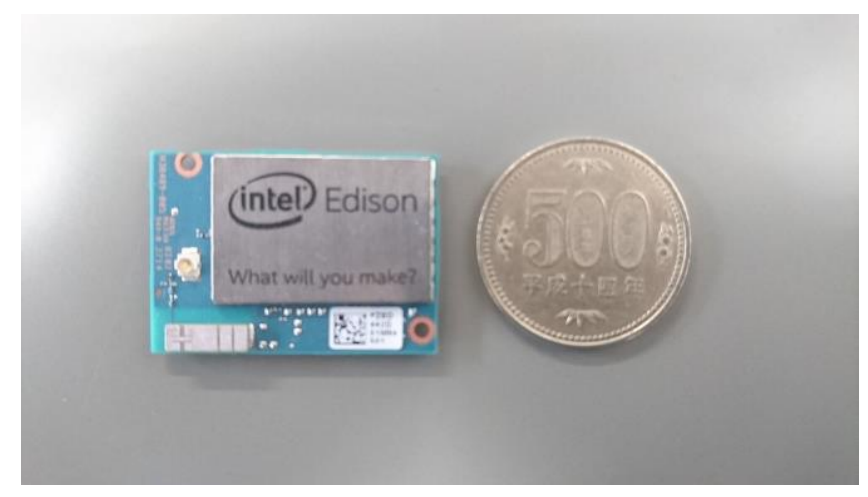

Fig. 8. Main processor unit of Smart Glasses "Intel Edison", that size around 500 Yen coin 


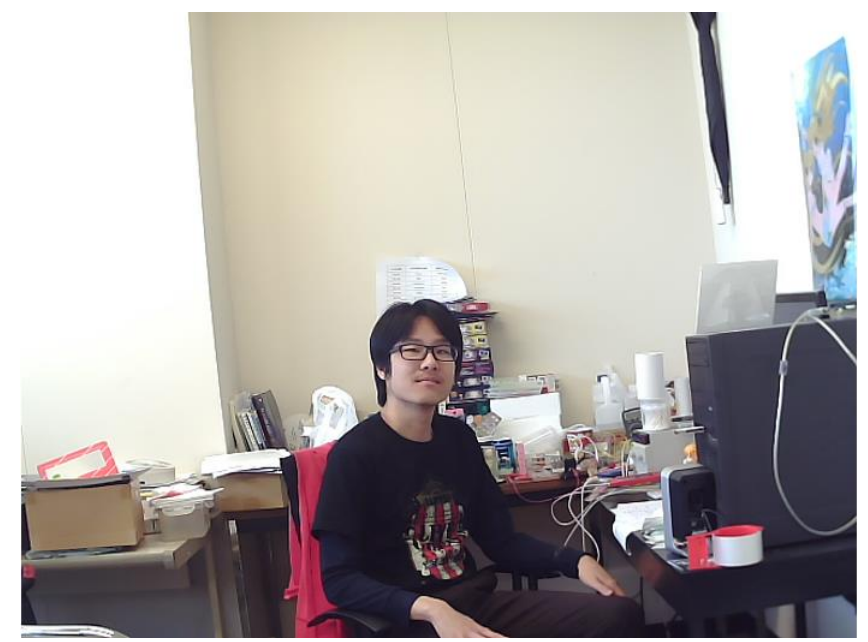

(1) Original Image

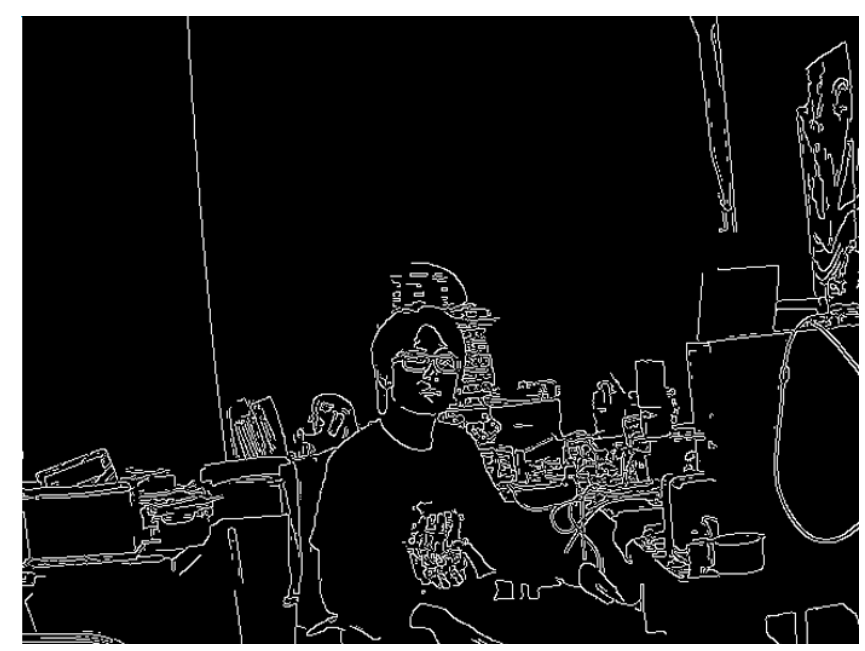

(2) Canny Edge Detection

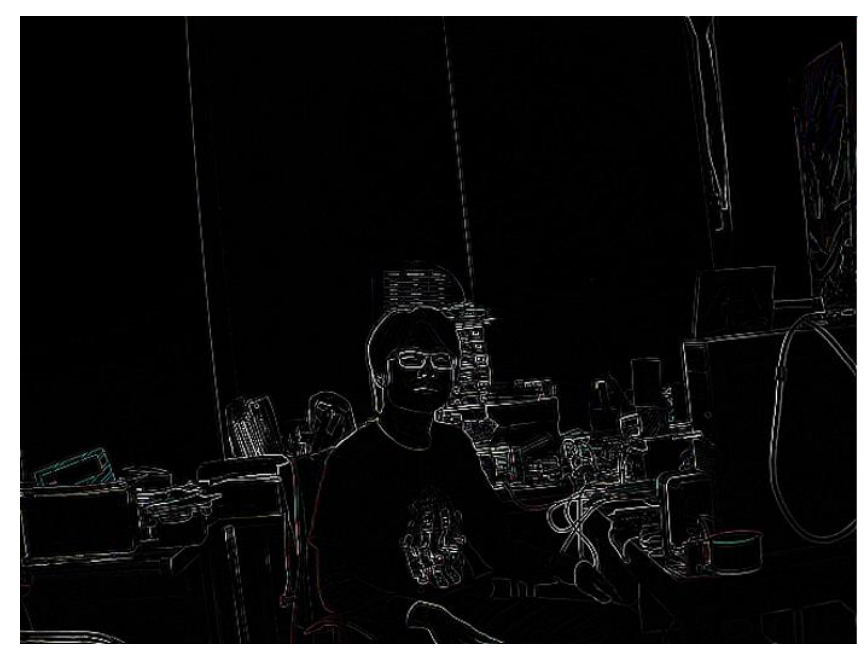

(3) Laplacian of Gaussian Edge Detection

Fig. 9, Comparison of (1) original image after used (2) Canny Edge Detection and (3) Laplacian of Gaussian

\subsection{Extracting information}

After our research environment preparation was finished, we installed OpenCV library on Intel Edison, and used OpenCV to develop image processing that can extract image's information data received from UVC camera. We wanted to extract image's information data that have less of unessential information. After main processor unit extracted image we can understand what it be. We chose Edge Detection algorithm to be our algorithm for extracting image's information data in our research. Edge Detection algorithm extracted the edge information from image, which means that original image from UVC camera will turn into black and white image. As shown in Fig. 9, the edge between black and white was object's border. Which made us knowing the shape of the objects. Edge Detection algorithm has many methods. Example; Canny Edge Detection ${ }^{(11)}$, Laplacian of Gaussian Edge Detection and others. The different of methods are about processing speed, contrast of shape of the objects. We chose Canny and Laplacian of Gaussian Edge Detection, and had test about processing speed. The Laplacian of Gaussian did the processing speed better than Canny, but Canny had contrast of shape of the objects better than Laplacian of Gaussian, as shown in Fig. 9, it is show how different of result the two method were. At the first we made main processor unit that can capture image, and extract information of image by using Edge Detection algorithm one by one. Then we made it can doing further more than that. In addition, main processor unit can extract information of image in real time.

\subsection{Resizing image}

We got extracted information image by Edge Detection algorithm, then we needed to make the extracted image to be smaller than it was. We need to resize image, because if the image size is too big, it will cause problem in others process of Smart Glasses. Furthermore, according from Noel's research result, the patient can recognize the sent image that have about 625 pixels. So that mean at first, we did not need to make it big or having too much information. In order to made image smaller, we used image processing to resize image from original resolution ( $480 \times 360$ pixel) to $60 \times 60$ pixel to see the result of the method. Then, we resized image from original size to $25 \times 25$ pixel. The image processing that we used is Geometric Image Transformation function on OpenCV ${ }^{(7)(8)(9)}$. 


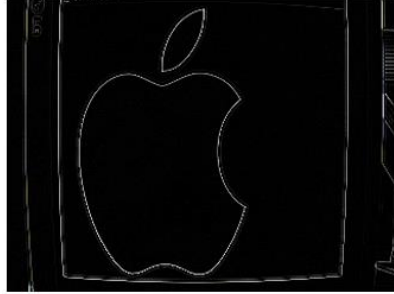

$480 \times 360$ Pixel

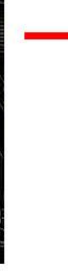

\section{$60 \times 60$ Pixel}

$25 \times 25 \mathrm{P}$

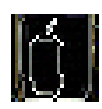

25 x 25 Pixel (True Scale)

Fig. 10, the result of resized image from original resolution to $60 \times 60$ pixel and then $25 \times 25$ pixel. At the bottom of figure is resized image true scale resolution $25 \times 25$ pixel that human eye can see in computer

Geometric Image Transformation function will not change image information or content, but it will deform pixel grid and map to destination grid image. For each pixel (x, y) of destination image, the Geometric Image Transformations function will calculate coordinates of corresponding donor pixel from image source and then it will copy the pixel value as show on equation.

$$
\operatorname{dst}(x, y)=\operatorname{src}\left(f_{x}(x, y), f_{y}(x, y)\right)
$$

As show on Fig. 10, is the result of resized image from original resolution ( $480 \times 360$ pixel) to $25 \times 25$ pixel.

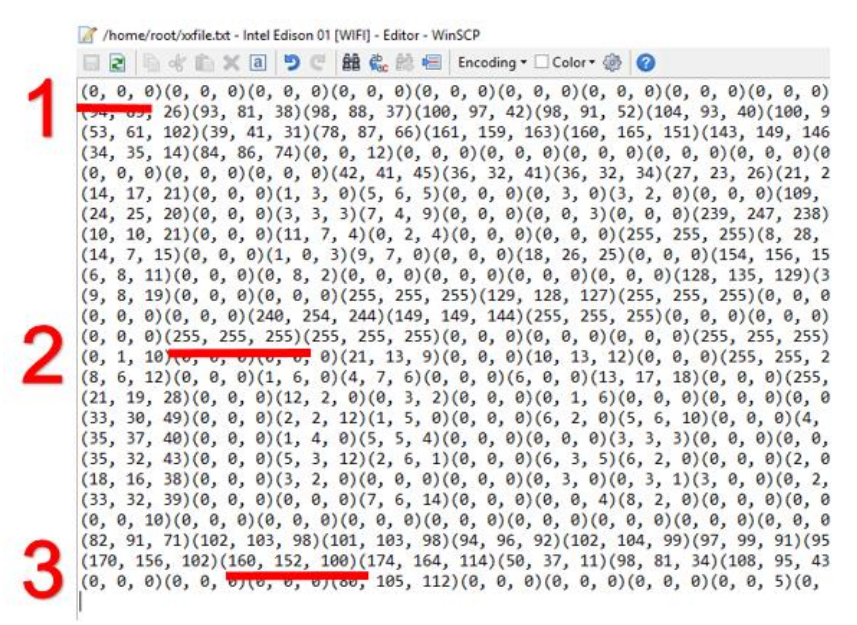

Fig. 11, Extracted pixel value of resized Laplacian of

Gaussian Edge Detection image. Red line number 1

$(0,0,0)$ mean black color pixel, number $2(255,255,255)$ mean white color pixel and number $3(160,152,100)$ and other values mean others color pixel (error)

\subsection{Converting image}

In this part, we got resized image from resizing image method. After that we need to convert resized image into binary text file to get each line of pixel value. Digital image is numeric representation of a two dimension image. The digital image may be raster or vector image that fixed by resolution of image. Raster image or bitmapped image, in image contain the value of pixel. Sorted in each line and column that make us seeing picture or shape. Main processor unit used image processing to read the each line and column of resized image, extracted pixel value of each line and column and wrote pixel value to binary text file. As shown in Fig. 11, in case of using Laplacian of Gaussian method in Edge Detection algorithm, main processor unit should get Red Blue Green (RGB) value like $(0,0,0)$ that means black color pixel and $(255,255,255)$ that means white color pixel. However we got others pixel value from resized image that used Laplacian of Gaussian Edge Detection as shown in Fig. 11. Because Laplacian of Gaussian method did not write out black and white color absolutely, it had error processing when extracted and represented image's objects. We added function into main processor unit to normalized each value pixel into one digit value, $(0,0,0)$ to $0,(255,255,255)$ to 1 . Furthermore, we fixed others pixel value that were not black or white by normalized it to white color pixel 1. Because the error pixel value is the white color pixel, they were not black color pixel. We normalized extracted pixel value of image because the data content on binary text file can be transmit to Artificial Eye with no complexity. After main processor unit got binary text file that data content of it was normalized, it will send this data content to Artificial Eye. Artificial Eye can use that data content to create electric current signal designed that will design by us.

\section{Conclusions}

Our research is designing and developing preprocess of Visual Prosthesis named Bionic Eyes. Bionic Eyes consists of two parts: Smart Glasses and Artificial Eye. In the present prototype Smart Glasses can receive image from camera and extract necessary information using image processing algorithm, resizing image and converting image in real time. It could be carried to use everywhere because we had mounted lithium-ion battery on it.

Our Future tasks are designing and developing Artificial Eye's circuit and energy transmitter part of Smart Glasses and receiver part of Artificial Eye. 


\section{Acknowledgment}

The authors thank Prof.Dr. Kei Eguchi of Fukuoka Institute of Technology for providing Bionic Eye's research material.

\section{References}

(1) Lei Zhao, Kaijie Wu, Xinyu Chai, Chaochen Gu, et al: "Image Processor for Visual Prosthesis Based on ARM", Biomedical Engineering and Informatics, PP.592-596, 2014

(2) N.R. Srivastava, P.R. Troyk, G.Dagnelie, D.Bradley : "Test Setup for Supporting Human Implantation of Intracortical Visual Prosthesis Device", $3^{\text {rd }}$ International IEEE EMBS Conference on Neural Engineering, PP.442-445, 2007

(3) James D. Weiland, Mark S. Humayun : "Visual Prosthesis", Proceeding of the IEEE, Vol. 96, No.7, PP.1076-1084, 2008

(4) Mingjie Sun, Kaijie Wu, Pengjia Cao, Xinyu Chai, Qiushi Ren : "Discrepancy of Electrical Evoked Potentials by Different Spatial-Temporal Stimulations of Optic Nerve Using Penetrating Electrode Array", IEEE/ICME International Conference on Complex Medical Engineering, PP.1293-1297, 2007

(5) Ying Zhao, Jia Wang, Xinyu Chai, Qiushi Ren : "Microstimulator Design for Visual Prosthesis based on Optic Nerve Stimulation", 2006 International Symposium on Biophotonics, Nanophotonics and Metamaterials, PP.14, 2006

(6) Jiajia Wang, Pengjia Cao, Liming Li : “A Preliminary Simulation Study of Virtual Channel Generated by Penetrating Optic Nerve Electrical Stimulation", BioMedical Engineering and Informatics, PP.522-526, 2014

(7) Borgefors, Gunilla : "Distance transformations in digital image", Comput. Vision Graph. Image Process.34 3, PP. 344-371, 1986

(8) Felzenszwalb, Pedro F. and Huttenlocher, Daniel P. : "Distance Transforms of Sampled Functions", 2004

(9) OpenCV Team : “OpenCV Reference Manual”, 2014

(10) Intel : "Intel Edison Module Hardware Guide”, PP.7-28, 2014

(11) John Canny : “A Computational Approach to Edge Detection", IEEE Transactions on Pattern Analysis and Machine Intelligence, Vol. PAMI-8, No.6, PP.680-698, 1986 